Helgoländer wiss. Meeresunters. 17, 108-125 (1968)

\title{
Über die hydrographische Struktur der Deutschen Bucht im Hinblick auf die Verschmutzung in der Konvergenzzone
}

\author{
ERICH GOEdECKE \\ Deutsches Hydrographisches Institut, Hamburg
}

\begin{abstract}
On the hydrographical structure of the German Bight in regard to the pollution in the zone of convergency. It seems necessary to study the hydrographic structure of the German Bight (southern North Sea) in order to be able to understand the significance of the convergency zone for the pollution in this area. The hydrographic picture obtained is based on $\mathrm{t}^{0} \mathrm{C}, \mathrm{S} \%$ and water current measurements. Dynamically, the convergency is a frontal zone in which two different bodies of water meet each other and are stratified. The water of the North Sea with its higher specific weight streams underneath the lighter coastal water. The horizontal and vertical distribution of density in this area creates irregular durations of high and low tides. Generally, low tides and their current run longer than high tides and their current. Data on the non-periodical rest current, based on distributions of S\%o and density $(\sigma t)$, have been recorded; its speed is higher on the north Frisian than on the east Frisian side. The horizontal and vertical distributions of temperature and salinity in the German Bight cause strong $t^{0} \mathrm{C}$ - and $\mathrm{S} \%$ gradients within the zone of convergence as well as pronounced seasonal differences. The convergency zone is important for the metabolic budget of this area, as it is likely that there exist close hydrographical, chemical, biological and geological interrelations. In springtime this area is oversaturated by oxygen due to intense plankton production. Moreover, it became evident that the convergency zone, with its high salinity gradient, forms an area in which several nutrient components like chalk, nitrate, phosphate, silicate (turbidity factor) accumulate. It was indeed possible to discriminate between different water bodies on the basis of the distributions of these nutrients.
\end{abstract}

\section{EINLEITUNG}

Wenn man die letzten 50 Jahre überblickt, so haben fast in jedem Jahrzehnt bestimmte Probleme der Nordseeforschung im Vordergrund gestanden. In den 20er Jahren waren es die Kohlensäurefaktoren des Meerwassers (vgl. hier auch die Ostseeforschung), in den 30er und 40er Jahren die Untersuchungen der Minimunstoffe (Nährstoffkomponenten), in den 50er Jahren die Produktivität des Meeres, und in diesem Jahrzehnt steht im Vordergrund die Verschmutzungsfrage in der Nordsee. Alle diese hier genannten meereskundlichen Probleme sind nicht endgültig gelöst worden. Frühere Fragestellungen werden mit heutigen, modernen Problemstellungen verknüpft, um Antworten auf diese Fragen zu erhalten. 
Im vorliegenden Fall ist die Aufgabe gestellt worden, in möglichst zusammenfassender Weise über die Ergebnisse zu berichten, welche das Vorhandensein einer Konvergenzzone innerhalb der Deutschen Bucht befürworten und darüber, welche Bedeutung diese Vermischungszone in den Fragen der Verschmutzung dieses Teils der Nordsee spielen könnte.

Um die Bedeutung dieser hydrographischen Erscheinung in der Deutschen Bucht verstehen zu können, ist es notwendig, sich ein zusammenfassendes Bild von der hydrographischen Struktur dieses Meeresraumes zu machen. Dieses hydrographische Bild über den Aufbau der Deutschen Bucht wird vornehmlich auf Grund der in den letzten fünf Jahrzehnten vorhandenen Temperatur-, Salzgehalts- und Strombeobachtungen sowie der daraus gewonnenen Ergebnisse und Erkenntnisse in diesem Raum der südöstlichen Nordsee geformt.

\section{DIE KONVERGENZ DER DEUTSCHEN BUCHT}

Die Konvergenz der Deutschen Bucht ist dynamisch bedingt (ZoRell 1935). Sie zeichnet sich an der Oberfläche wie in der Tiefe des Meeres durch besonders starke Temperatur- und Salzgehaltsgradienten aus, welche vertikal gesehen eine ausgeprägte thermohaline Schichtung zur Folge hat. Die Konvergenz der Deutschen Bucht ist daher als eine Zone intensiver Mischung von mindestens zwei hydrographisch verschiedenen zu- und aufeinanderströmenden Wasserkörpern aufzufassen (Kalle 1937, 1943). Nach der Definition von Kalle versteht man unter Wasserkörper folgendes: „Ein Wasserkörper ist eine Wassermasse, welche sich auf Grund ihrer gemeinsamen Herkunft oder infolge einer gleichförmig auf sie wirkenden Kraft als deutlich zusammengehörige Einheit betrachten läßt. Kennzeichnen lassen sich solche Wasserkörper auf Grund ihres gleichförmigen Verhaltens in bezug auf physikalische, chemische oder biologische Eigenschaften." Für die Unterscheidung von Wasserkörpern in horizontaler und vertikaler Hinsicht sind vor allem solche hydrographischen Faktoren am besten geeignet, die gegenüber äußeren Einflüssen im Meere große Stabilität aufweisen. Zwei verschiedene Wasserkörper - im vorliegenden Fall sind es in der Deutschen Bucht das salzreichere und damit spezifisch schwerere westliche Nordseewasser sowie das salzärmere und damit spezifisch leichtere Küstenwasser - treffen aufeinander und bilden eine Grenzfläche heraus, die trotz Turbulenz und Vermischung erhalten bleibt, wenn nur auf beiden Seiten derselben immer wieder neue Wassermassen der charakteristischen Art herangeführt werden. In der Natur werden sich statt Konvergenzlinien in den meisten Fällen bereitere Konvergenzzonen herausbilden, in denen starke Vermischungen der Wasserkörper unter mehr oder weniger intensiver Einwirbelung stattfinden.

\section{DER 'THERMOHALINE AUFBAU}

Auf Grund einer gemeinschaftlichen Arbeit über die Salzgehaltsverhältnisse der Nordsee für die Periode 1902 bis 1954 (Goedecke, Smed \& TomczaK 1967) wird an zwei Salzgehaltskarten für den Monat Juni sowie für die oberflächennahe Schicht 7,5 m 
und für den Boden vorgelegt (Abb. 1 und 2). Diese Karten zeigen die Salzgehaltsverteilung im großen Rahmen der gesamten Nordsee. Mehr trichterförmig in der Tiefe als an der Oberfläche schiebt sich das Nordseewasser in die Deutsche Bucht hinein. Dabei ist schon auf dieser Ubersichtskarte trotz des kleinen Maßstabs zu erkennen, daß das

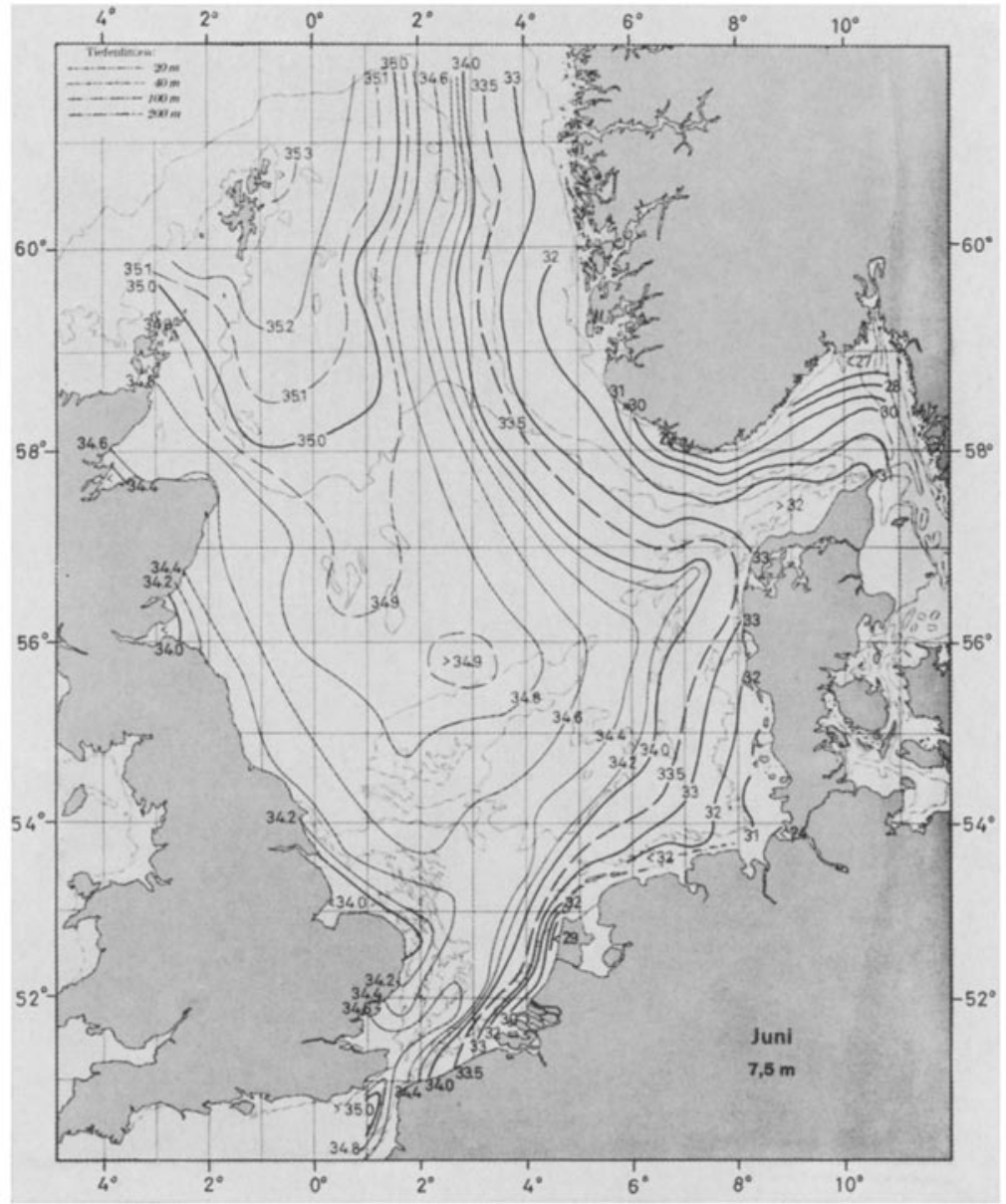

Abb. 1: Mittlere Salzgehaltsverteilung in oberflächennahen Schichten $(7,5 \mathrm{~m})$ der Nordsee. (Nach Goedecke, SMed \& Tomczak 1967)

Tiefenwasser der Nordsee weiter östlich reicht als an der Oberfläche. Außerdem weisen die westlichen Ausbuchtungen der Isohalinen auf das Vorhandensein des Küstenwassers hin. Dies sind Beispiele von Mittelwertskarten. Welche Begrenzungen man bei der Interpretation von Karten mit mittleren Salzgehaltsverhältnissen ansetzen muß, hat vor allem WatTENBERg (1941) bei seinen Untersuchungen der Grenzen zwischen Nordund Ostseewasser gebührend hervorgehoben: mittlere Verhältnisse brauchen in der Natur nicht vorzukommen. Es ist daher angebracht, in diesem Zusammenhang auf 
singuläre Verhältnisse hinzuweisen. Monatliche mittlere Salinitäts-Darstellungen, wie beispielsweise im Mai 1933 (GOEDECKE 1941), weisen auf starke Verwirbelung an der Oberfläche und auch am Boden hin (Abb. 3 und 4). Wie bereits in den vorgehenden großen Nordseekarten leicht angedeutet, dringt das Tiefenwasser der Nordsee weiter

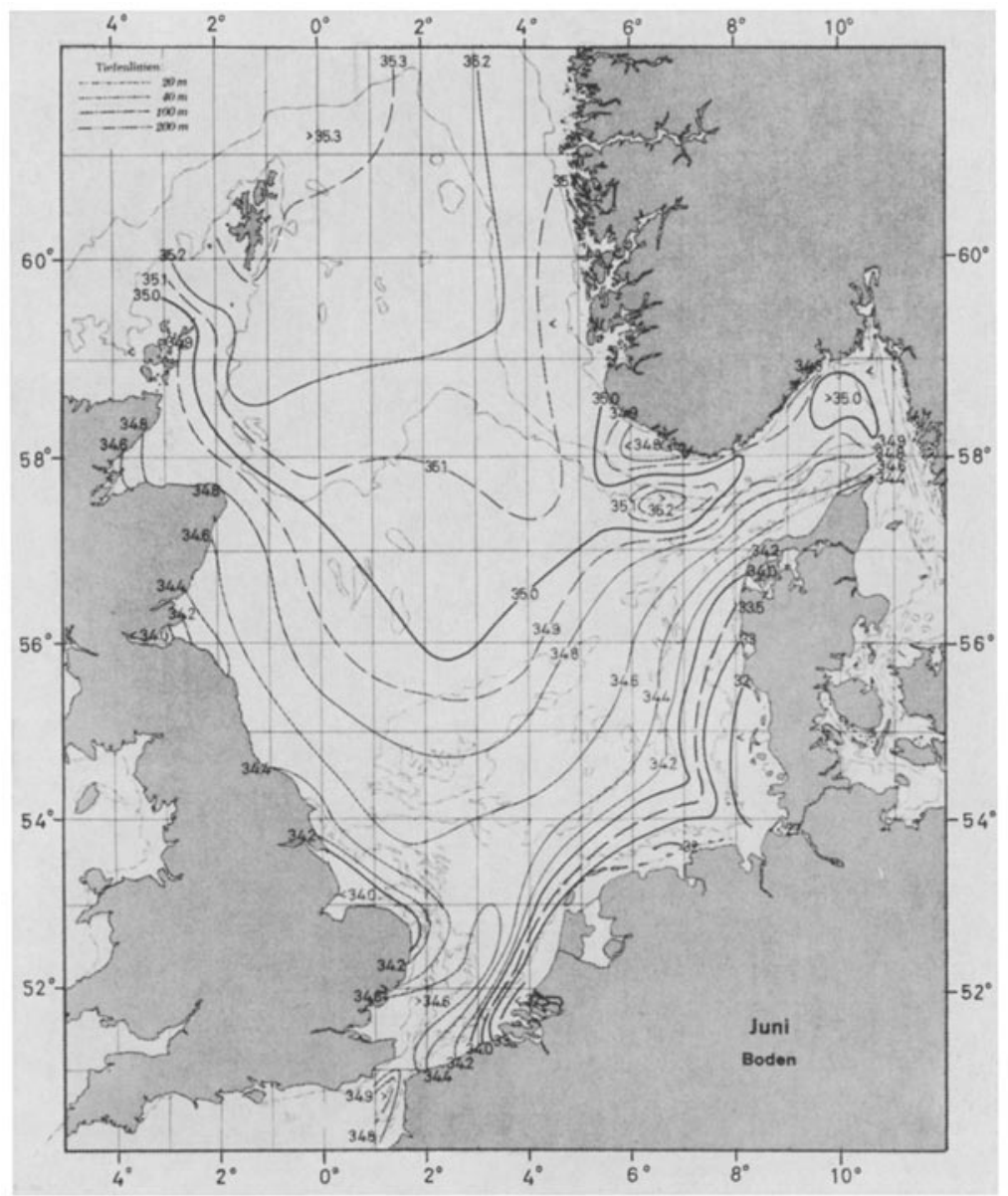

Abb. 2: Mittlere Salzgehaltsverteilung am Boden der Nordsee. (Nach Goedecke, SMed \& ToMcZak 1967)

in die Mündungstrichter von Elbe und Weser ein als das Oberflächenwasser. Die ostfriesische Küstenregion zeigt hinsichtlich des Salinitäts-Verhaltens bemerkenswerte Unterschiede gegenüber der nordfriesischen Küstenregion auf. Gedrängte Schaarung der Isolinien an der ostfriesischen Seite und aufgelockerte Ausbildung der Isolinien entlang der nordfriesischen Seite. In der Mitte der Deutschen Bucht jedoch sind innerhalb einer ausgeprägten von NW nach SO reichenden Zone starke Salzgehaltsgradienten auf kurzem Raum ausgebildet. Als Ergänzung hierzu noch zwei Beispiele für Salzgehalts- 
Untersuchungen kürzerer Perioden. Für die 2. März-Dekade 1935 und die 2. FebruarDekade 1936 (Abb. 5 und 6) ist die Vermischung der Konvergenzzone noch stärker ausgeprägt als in den vorhergehenden Darstellungen (GOEDECKE 1958a, b).

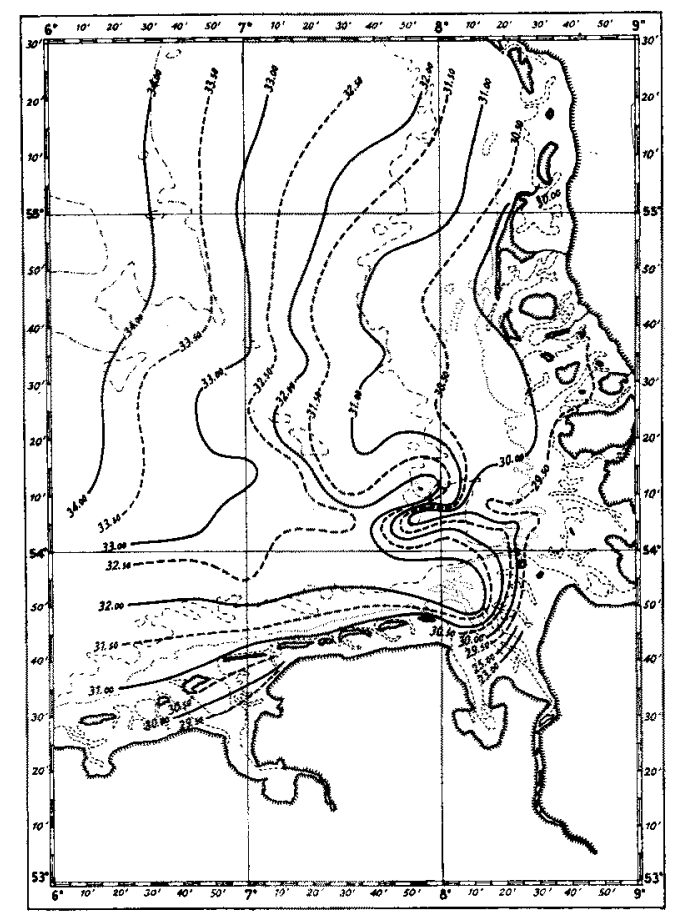

Abb. 3: Mittlere Salzgehaltsverteilung im Oberflächenwasser, 12. bis 23. Mai 1933. (Nach GOEDECKE 1941)

\section{DIE OBERFLACHENSTROME}

Nach den vorhergehenden Darlegungen ist die Konvergenz der Deutschen Bucht dynamisch gesehen eine Frontalzone, in welcher zwei verschiedene Wasserkörper aufeinandertreffen und sich überlagern. Das schwerere Nordseewasser schiebt sich unter das leichtere Küstenwasser.

In der Nordsee werden die periodischen Gezeitenströme von den sogenannten unperiodischen Restströmungen, welche die eigentlichen Wasserversetzungen in diesem Meeresgebiet verursachen, überlagert (KaLLE 1949). Denn die Gezeitenströme für sich bestehen aus periodischen Schwingungen um einen Punkt. Die überlagerten Restströme jedoch rekrutieren sich aus dem sogenannten Küsteneffekt, nämlich Wasserständen und anomaler Größe der Abflußmengen aus den Flußmündungstrichtern sowie der Dichteverteilung der Deutschen Bucht und dem Einfluß der Wetterlagen auf die hydrographischen Verhältnisse. Sie sind für die Verfrachtung insbesondere von ungelösten Partikelchen und biologischen Kleinlebewesen verantwortlich zu machen. Diese Restströme 


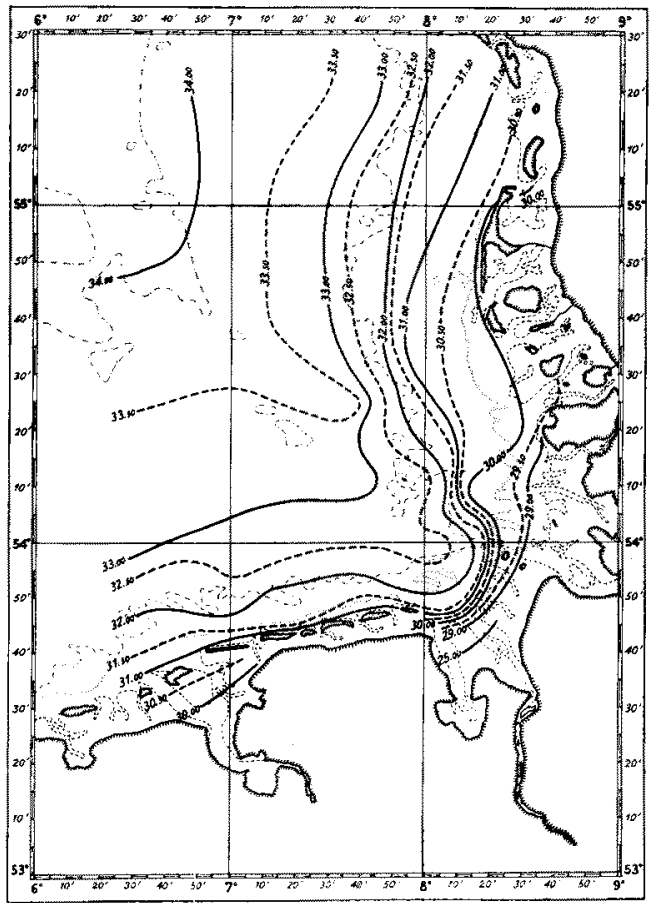

Abb. 4: Mittlere Salzgehaltsverteilung im Bodenwasser, 12. bis 23. Mai 1933. (Nadh GOEDECKE 1941)

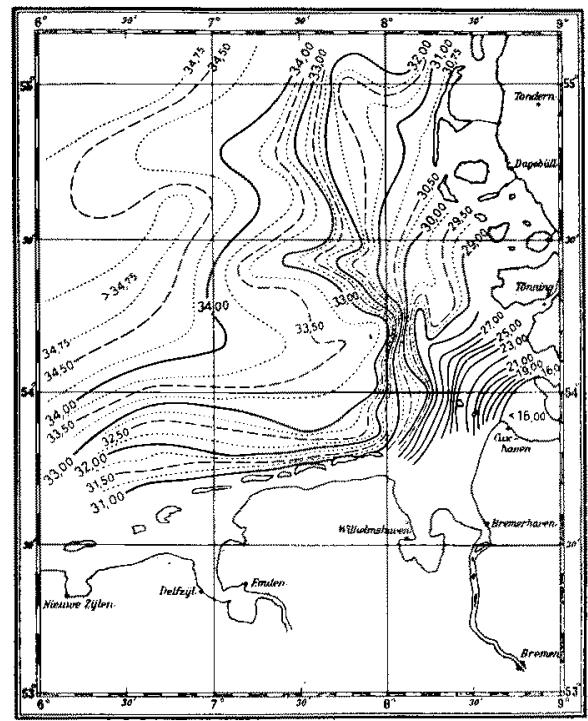

Abb. 5: Salzgehaltsverteilung an der Oberfläche während der zweiten März-Dekade 1935. (Nach GOEDECKE 1958a) 


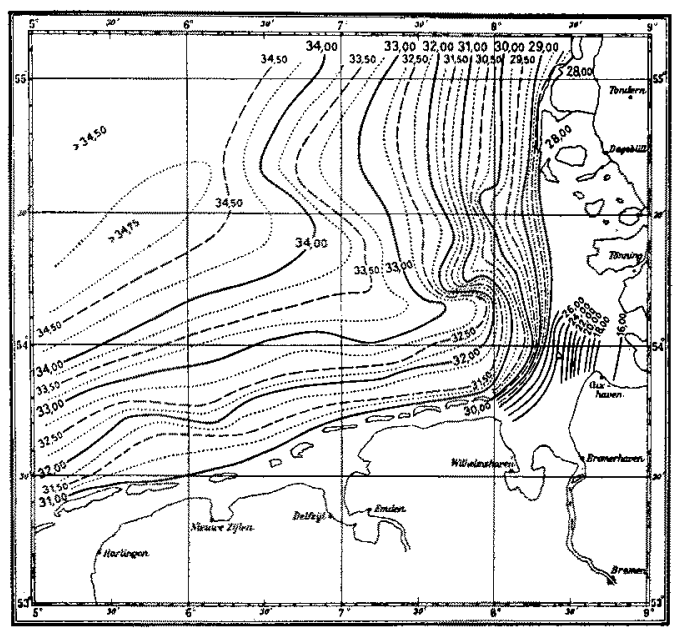

Abb. 6: Salzgehaltsverteilung an der Oberfläche während der zweiten Februar-Dekade 1936. (Nach GOEDECKE 1958a)

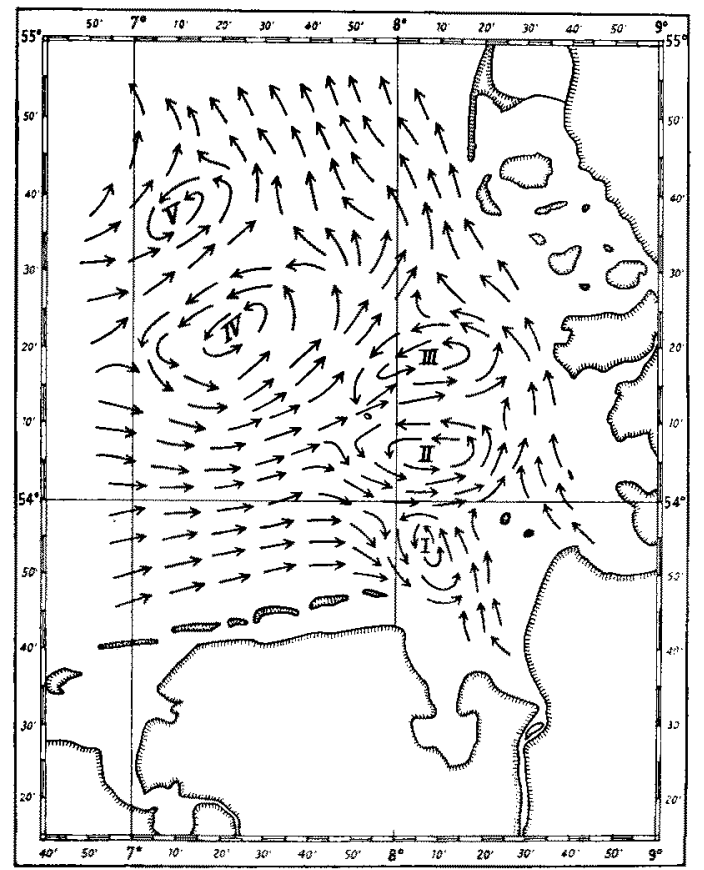

Abb. 7: Wasserversetzungen (Restströmungen) an der Oberfläche der Deutschen Bucht. Grundlage: synoptische Salzgehaltskarte vom 23. bis 25. Juni 1930. (Nach Gozdecke 1939) 
müssen hinsichtlich der Frage der Verschmutzung des Meerwassers der Deutschen Bucht als bedeutender Faktor herausgestellt werden. Da Strombeobachtungen nur in Teilgebieten der Nordsee bis Ende der 30 er Jahre vorlagen, hatte man auch nur großzügige Vorstellungen über die Art und den Verlauf von unperiodischen Wasserversetzungen innerhalb der Deutschen Bucht. WENDICKE (1913) und ZoRELL (1935) hatten an Hand von Salzgehaltskarten der Oberfläche, zum Teil auch für die Tiefe, festgestellt, daß die un-

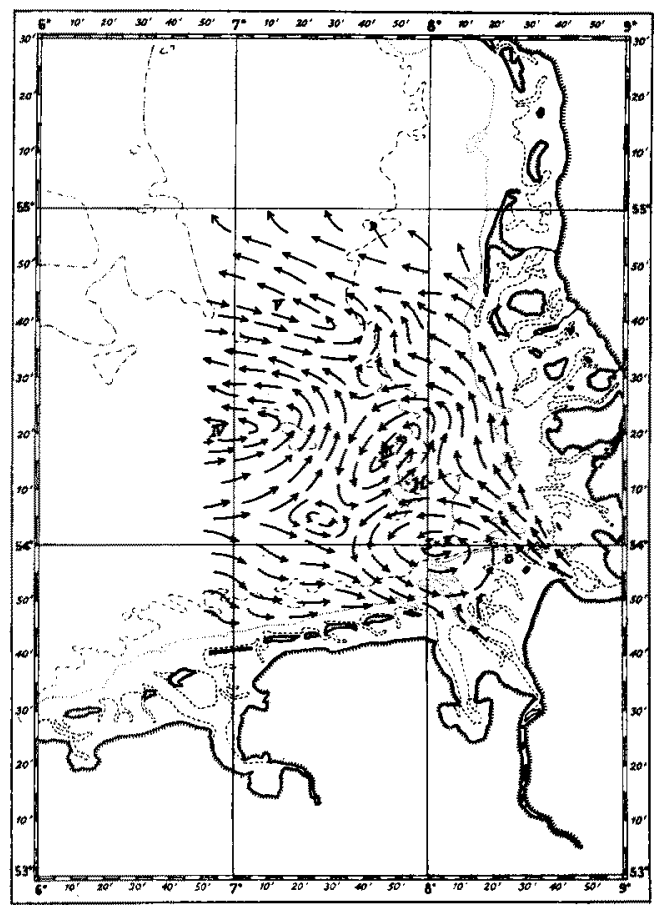

Abb. 8: Wasserversetzungen (Restströmungen) aus der Oberfläche der Deutschen Bucht. Grundlage: Mittlere Salzgehaltsverteilung vom 12,- 23. Mai 1933. (Nach Goedecke 1941)

periodischen Wasserversetzungen innerhalb der Deutschen Bucht sich durch einen großräumigen zyklonalen Wirbel bemerkbar machen. Durch Analyse von vielen S\%/00-Karten auf Grund von Einzelfahrten ist mit Hilfe der Methode von BöHNEckE (1922) versucht worden, wie für die gesamte Nordsee auch für die Deutsche Bucht derartige Stromkarten darzustellen. An Hand von zwei Beispielen (Abb. 7 und 8) werden auf Grund der S\%00-Verteilung von Juni 1930 und Mai 1933 Darstellungen von unperiodischen Wasserversetzungen in Erinnerung gebracht (GoEDEcke 1939, 1941). Innerhalb des großen zyklonalen Wirbels sind entlang der Konvergenzzone bis zu fünf Teilwirbel vorhanden, welche deutlich die starke Vermischungszone herausstellen. Berechnungen der Restströme aus direkten Strombeobachtungen in der Deutschen Bucht werden zeigen, ob die Vorstellungen von unperiodischen Wasserversetzungen wie in diesen Bildern 


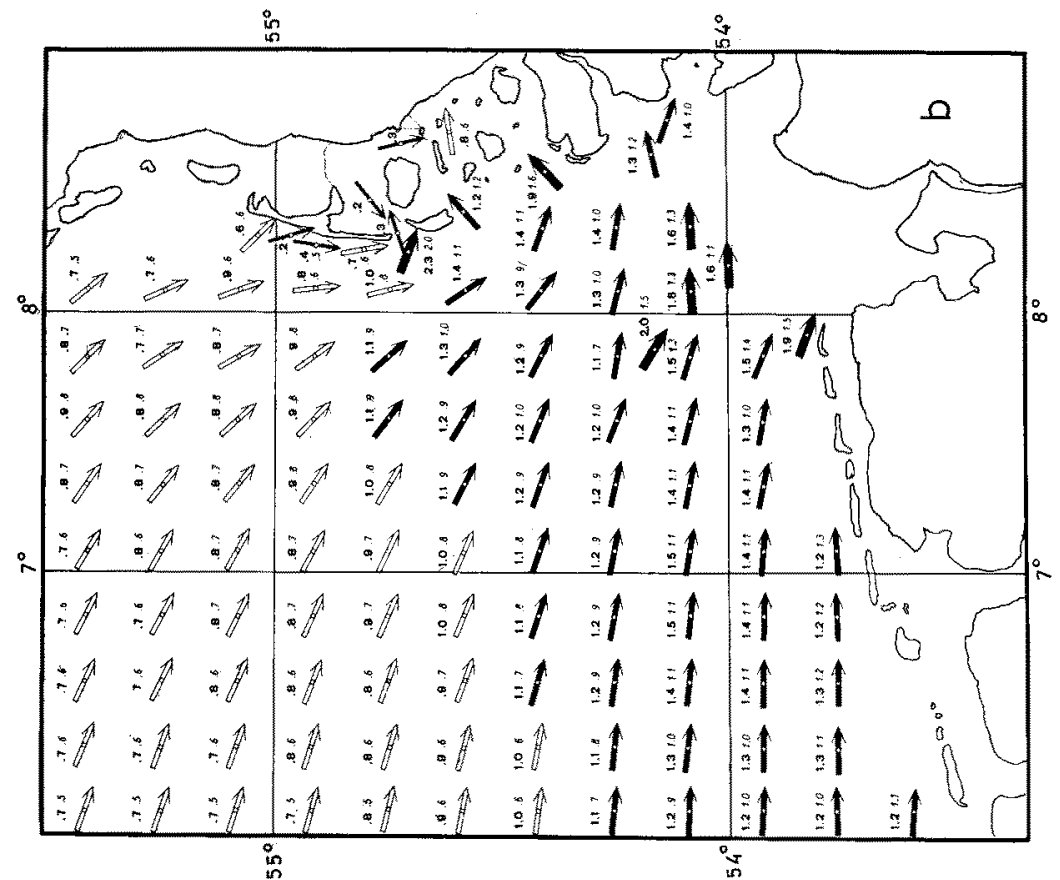

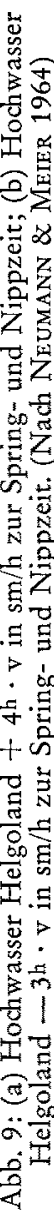

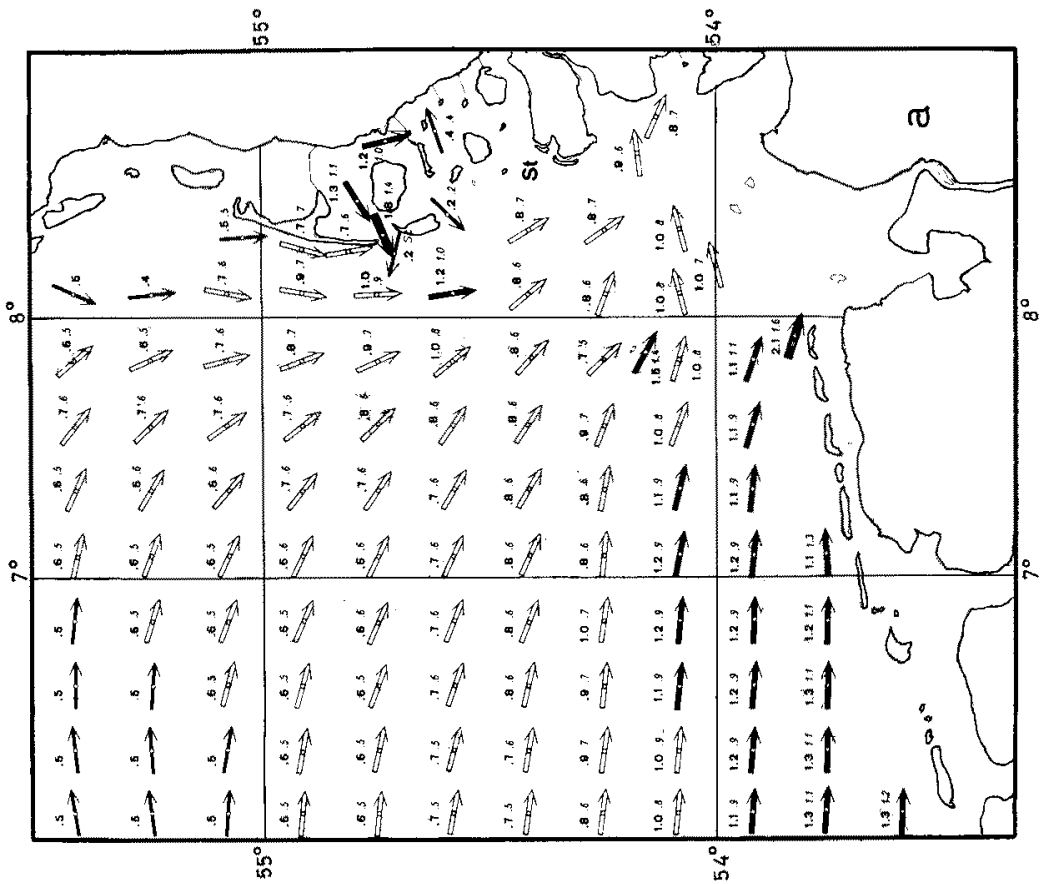


zurecht bestehen. Berechnungen von Stromversetzungen (des Massentransportes) in der Oberflächen- und Bodenschicht auf Grund der Dichteverteilung ergaben bisher einen Dichtegradientstrom von der Größenordnung $10 \mathrm{bis} 15 \mathrm{~cm} / \mathrm{sec}$ vor den Mündungstrichtern der Elbe und Weser sowie Grenzwerte von 4 bis $10 \mathrm{~cm} / \mathrm{sec}$ in der Konvergenzzone. Diese Werte sind aber höher als in den angrenzenden Bereichen. Dabei zeigt die nordfriesische Küstenregion wiederum höhere Geschwindigkeitswerte als die ostfriesische Region.

Frühere Untersuchungen von MöLler (1933) und neuere Untersuchungen von Neumann \& Meler (1964) weisen darauf hin, daß die Dichteverteilung in der Deut-

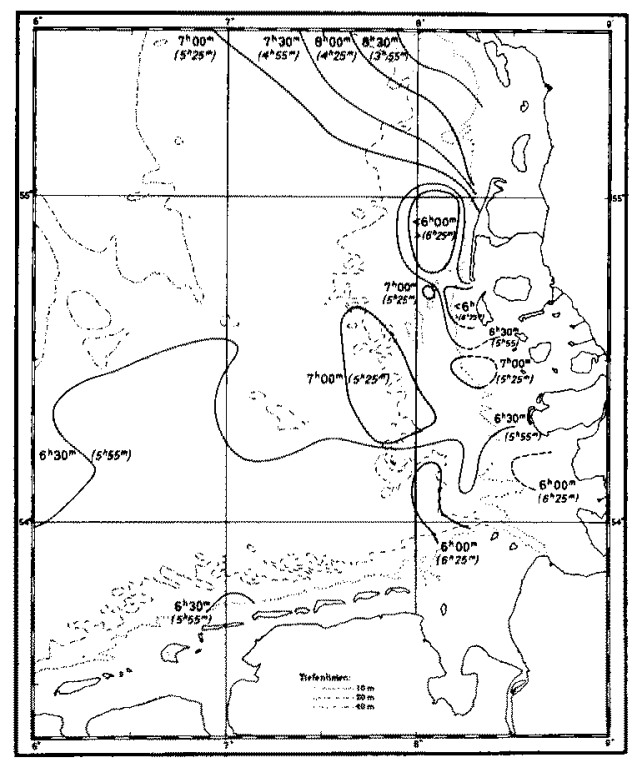

Abb. 10: Dater des Ebbstromes (Flutstromes). (Nach Neumann \& MeIER 1964)

schen Bucht eine Asymmetrie in der Dauer von Flut und Ebbe sowie in derjenigen von Flut- und Ebbstrom verursacht. Im großen und ganzen gesehen läuft die Ebbe beziehungsweise der Ebbstrom länger als die Flut beziehungsweise der Flutstrom. Außerdem sind bemerkenswerte Unterschiede zwischen der Steigdauer und Falldauer vorhanden. Nach Möller (1933) ist Steigdauer die Differenz zwischen Eintritt des Niedrigwassers und dem des folgenden Hochwassers. Die Abbildungen 9 und 10 kennzeichnen die Situation innerhalb der Gezeitenströme. Das Küstenwasser bewirkt eine Verlängerung der Ebbstromdauer. Noch viele Einzelheiten könnte man aus diesen neuesten Ergebnissen entnehmen. Es muß in diesem Zusammenhang auf die Veröffentlichung von Neumann \& Meier (1964) hingewiesen werden. Stets zeigt sich, welch überragende Rolle das Küstenwasser, besonders die Ausbreitung des Oberwassers aus den großen Stromtrichtern von Elbe und Weser im Wasserhaushalt der Deutschen Bucht spielt. 


\section{DIE THERMOHALINE SCHICHTUNG}

Die Ausbreitung des Küstenwassers aus den Flußmündungen zeigt Abbildung 11 (Goedecke 1952). Sie gibt die S\%on-Schichtung im Frühjahr (April/Mai 1932) auf Grund der Differenz Boden minus Oberfläche wieder. Die Konvergenzzone stellt sich hier als eine von SO nach NW gerichtete Zone mit relativ hohen S\%on-Differenzen dar.

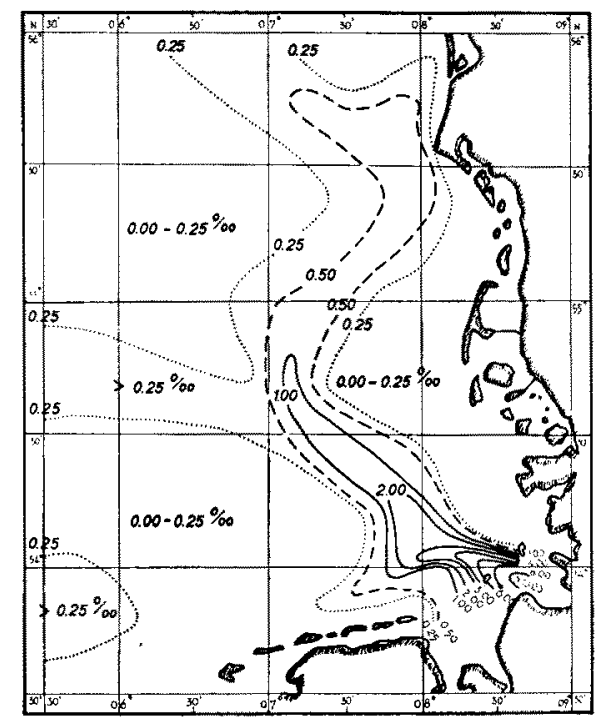

Abb. 11: S\%0-Schichtung in der Deutschen Bucht von April bis Mai 1932 auf Grund der Salinitätsdifferenzen an der Bodenoberfläche. (Nach GoEDECKE 1952)

Daß das Konvergenzgebiet nicht nur auf die Oberflächenschicht beschränkt bleibt, sondern auch die Tiefenschichten erfaßt, wird in den Abbildungen 12 bis 15 wiederzugeben versucht. Die thermohaline und Dichte- $\left(\sigma_{\mathrm{t}^{-}}\right)$Schichtung im Frühjahr wird wieder durch Differenz Oberfläche minus Boden ausgedrückt (GOEDECKE 1955). In der Konvergenzzone sind relativ hohe Unterschiede zwischen den hydrographischen Werten der Deckund Bodenschicht vorhanden.

Die bisherigen Querprofile in der Deutschen Bucht haben ergeben, daß in den Wintermonaten in der südöstlichen Nordsee Homothermie vorherrscht. Gute Durchlïftung und kräftige Durchmischung der Wasserkörper findet statt. Es kann aber vorkommen, daß bei langandauernden, ablandigen Winden sich eine thermische Sprungschicht zwischen dem kälteren Oberflächenwasser und dem wärmeren Bodenwasser bildet. Mit zunehmender Erwärmung und Wirkung des in anomaler Menge abfließenden wärmeren Küstenwassers in die Deutsche Bucht tritt von April bis Juli eine maximale thermische Schichtung ein. Die Tiefe dieser Sprungschicht liegt über flacherem Wasser in 5 bis $15 \mathrm{~m}$ und uber tieferem Wasser zwischen 15 und $20 \mathrm{~m}$. Die Zweiteilung - relativ warme Oberschicht und relativ kalte Bodenschicht - reicht von den tieferen Regionen der Deutschen Bucht etwa bis zu der 15- beziehungsweise 20-m-Tiefenlinie. 
Höher hinauf bis zu den Watten liegt die fast vollkommen homotherme Küstenwasserregion. Im August und September ist der Höhepunkt der thermischen Sprungschicht überschritten. Mit der zunehmenden Abkühlung nimmt die Intensität ab, bis der Winterzustand wieder erreicht ist.

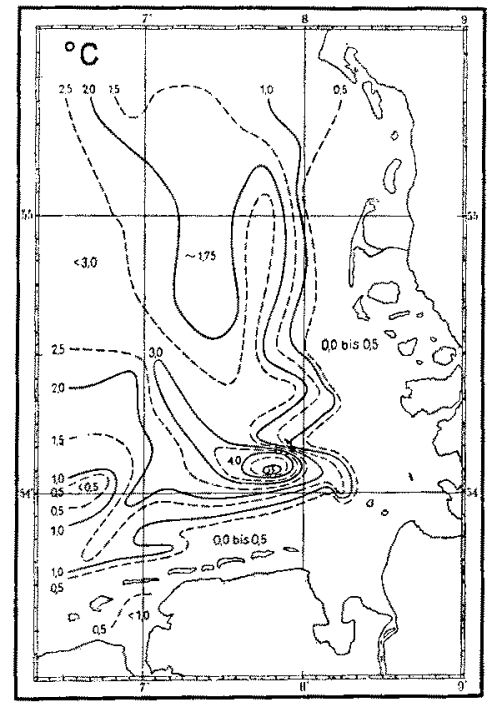

Abb. 12: Thermische Schichtung auf Grund der Temperaturdifferenzen Oberfläche minus Boden im Mai 1933. (Nach GOEDECKE 1955)
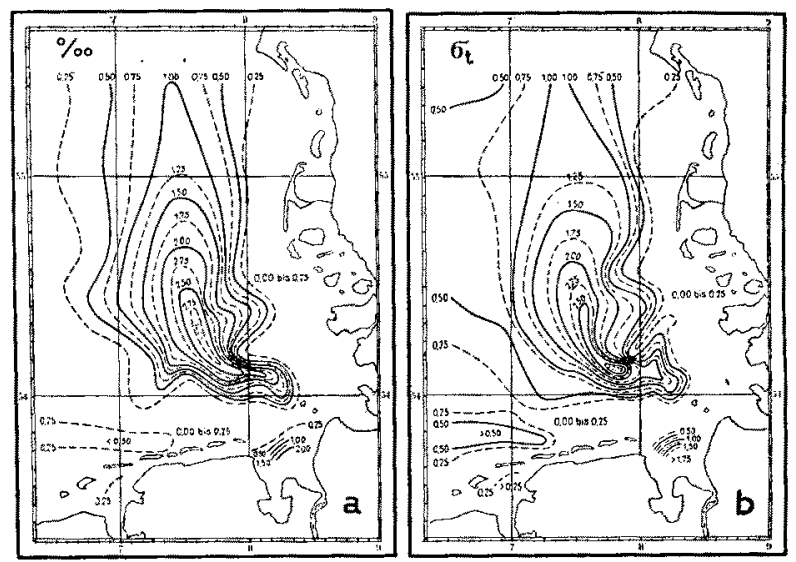

Abb. 13: (a) Haline Schichtung auf Grund der Salzgehaltsdifferenzen (Boden minus Oberfläche) im Mai 1933. (b) Dichte-Schichtung auf Grund der $\sigma_{t}$-Differenzen (Boden minus Oberfläche) im Mai 1933. (Nach Goedecke 1955)

Von einem entsprechenden Jahresgang in der vertikalen $\mathrm{S}^{0} \% 0^{-V}$-Verteilung kann nicht gesprochen werden. Jeder Wasserkörper - hier salzreiches Nordseewasser, dort salzarmes Küstenwasser - zeigt, für sich betrachtet, zu allen Jahreszeiten nahezu Homo- 


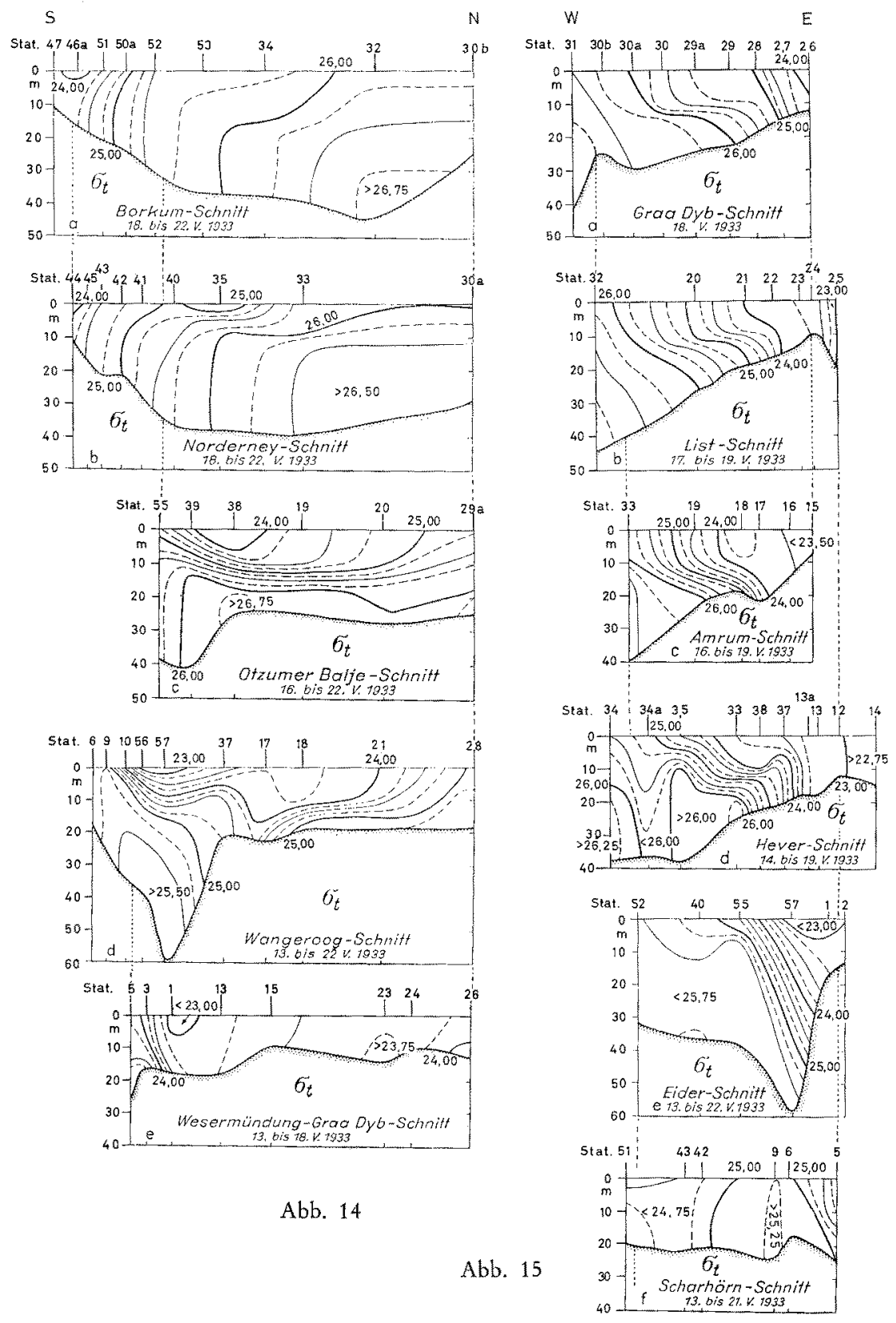

Abb. 14: Vertikale Dichte $\left(\sigma_{t}\right)$-Verteilung auf Schnitten senkrecht zur ostfriesischen Küste bei 2000 facher Ubertiefung (a bis c: 16. bis 22. Mai 1933; d bis e: 13. bis 22. Mai 1933). (Nach GOEDECKE 1935)

Abb. 15: Vertikale Dichte $\left(\sigma_{t}\right)$-Verteilung auf Schnitten senkrecht zur nordfriesischen Küste bei 2000 facher Obertiefung (a bis $f: 13$. bis 22. Mai 1933). (Nach GOEDECKE 1955) 
halinität. Nur in der Konvergenzzone, wo sie sich übereinanderschieben, herrscht zu allen Jahreszeiten mehr oder weniger starke haline Schichtung vor. Durch den anomalen Abfluß der Süßwassermengen während der Frühjahrsmonate wird von Mai bis Juli das Maximum der halinen Schichtung erreicht. Die Intensität und Ausdehnung der thermohalinen Sprungschicht während dieser Jahreszeit kann an Querprofilen senkrecht $z u$ den friesischen Küsten verfolgt werden. In Abbildungen 14 und 15 sind als Beispiele die vertikalen Dichte- $\left(\sigma_{t^{-}}\right)$Verteilungen im Mai 1933 dargestellt (GoEdecke 1955). Im Westteil der Deutschen Bucht ist ein fast einheitlicher Dichteaufbau vorhanden. Gute Durchmischung der Wasserkörper ist landwärts zu beobachten. Die Konvergenzzone ist nur im $\mathrm{N}$ angedeutet. Weiter östlich wird immer mehr das Konvergenzgebiet angeschnitten. Weit nach W ausholende Zungen von Küstenwasser und starke Verwirbelungen sind zu erkennen. Die an der nordfriesischen Seite angelegten Profile (Graa Dyb - Scharhörn) zeigen als gemeinsames Merkmal die Uberschiebung der beiden Wasserkörper der Deutschen Bucht. In den mittleren Schnitten ist die Dichtesprungschicht innerhalb der Konvergenzzone besonders deutlich ausgebildet.

Es ist immer wieder erstaunlich, wie trotz der geringen Wassertiefe und der zeitweise turbulenten Wasserbewegungen die Grenzfläche zwischen den beiden Wasserkörpern verschiedener Dichte erhalten bleibt. Ständige Zufuhr neuer Wassermassen aus den Wasserkörperzentren erhält eine stationäre thermohaline Sprungschicht.

\section{BEZIEHUNGEN ZWISCHEN HYDROGRAPHISCH-CHEMISCHEN UND MEERESBIOLOGISCH-GEOLOGISCHEN FAKTOREN}

Die Bedeutung der Konvergenzzone der Deutschen Bucht für den Stoffhaushalt dieses Meeresgebietes der Nordsee ist darin zu sehen, daß aller Wahrscheinlichkeit nach innige hydrographische, chemische, biologische und geologische Zusammenhänge vorhanden sind.

Auf Grund von Alkalinitätsuntersuchungen in der Deutschen Bucht (GoEDECKE 1936) ist man zu der Erkenntnis gekommen, daß auch die Sauerstoffverhältnisse innerhalb der Konvergenzzone von besonderer Wichtigkeit sind. Die beiden Ubersichtskarten mit der horizontalen Sauerstoffverteilung im Oberflächen- und Bodenwasser (Abb. 16 und 17) zeigen im Frühjahr die Besonderheit, daß in der Mitte der Deutschen Bucht eine breite Zone mit Sauerstoffübersättigung vorhanden ist. Diese erstreckt sich in der gleichen Richtung wie die Konvergenzzone (GoEDECKE 1941). Westlich und östlich dieser Zone hat man Werte unter $100 \%$ Sättigung beobachtet. Welche Erklärung hat man dafür? Der Temperaturfaktor kommt nicht in Frage. Temperaturerhöhung verursacht $\mathrm{O}_{2}$-Erniedrigung. Starke $\mathrm{O}_{2}$-Veränderungen in oberflächennahen Wasserschichten sind auf Tätigkeit assimilierender Lebewesen zurückzuführen. Durch anomalen Abfluß der Elbe- und Weseroberwassermassen werden ungeheure Mengen von Nährstoffen in die Deutsche Bucht befördert. Außerdem werden durch den Zusammenprall der Wassermassen in der Konvergenzzone weitere Nährstoffe gebildet und angereichert. Die Konvergenz ist ein Gebiet besonderer Planktonproduktion und -autolyse. Durch diese Nahrungsquelle der für das Phytoplankton lebensnotwendigen Minimumstoffe wird die Assimilation oder Photosynthese angeregt. Die Zunahme der Photosynthese äußert sich 
in einem Uberschuß von gelöstem Sauerstoff. Die Zone mit $\mathrm{O}_{2}$-Übersättigung fällt in gleicher Richtung mit derjenigen der Konvergenzzone zusammen. Das $\mathrm{O}_{2}$-Überschußgebiet reicht bis zum Boden und ist dort gegen die nordfriesische Küste orientiert. Das deutet wie bei der Salzgehaltsverteilung auf eine Schrägstellung der nach Osten einfallenden Konvergenz hin (Abb. 18).

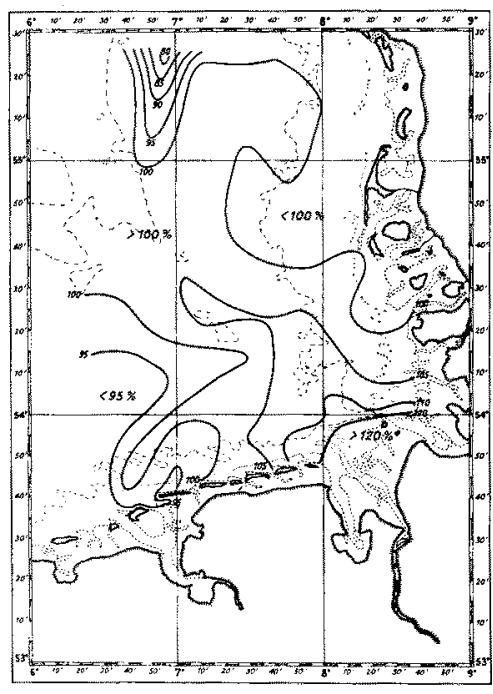

Abb. 16: Verteilung des relativen Sauerstoffgehalts im Oberflächenwasser vom 12. bis 23 . Mai 1933. (Nach GoEdecke 1941)

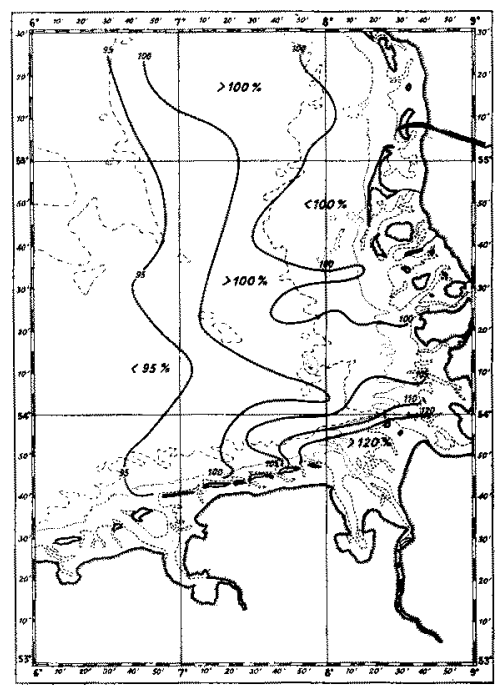

Abb. 17: Verteilung des relativen Sauerstoffgehaltes im Bodenwasser vom 12. bis 23. Mai 1933. (Nach Gobdecke 1941)

Auf Grund der Ergebnisse von Kalkgehaltsuntersuchungen in der Deutschen Bucht ist das Vorhandensein eines Konvergenzgebietes wahrscheinlich am besten bestätigt worden (GOEDECKE 1936). Es hat sich herausgestellt, daß die Konvergenz mit ihren starken Salzgehaltsgradienten ein Gebiet ist, in dem nicht nur vorwiegend anorganische Kalkausfällung und organische Kalkzerstörung stattfinden, sondern auch eine Produktionszunahme des kalkführenden Planktons ist. Das Gebiet mit anomalem Gehalt an suspendiertem Kalk fällt mit der Konvergenzzone zusammen. Weiterhin fällt diese Zone mit anomalem Kalkgehalt und diejenige der „Stationären Nord-Südmischwasserzone (Planktongemeinschaft: Kleine Peridinium-Arten) zusammen.

Weitere Zusammenhänge zwischen physikalischen und Nährstoff-Faktoren innerhalb der Konvergenzzone kann man nach Kalde (1932) in einer Zone mit Phosphatüberschuß erblicken. Die durch die Flußwassermassen ins Meer transportierten Süßwasserplanktonten verfallen im Konvergenzgebiet der Autolyse und reichern den P-Gehalt des Meerwassers an. Diese Anreicherung der Nährstoffe bildet wiederum die Grundlage für eine intensive Wucherung dort lebensfähiger Planktonten. Durch die systematischen Untersuchungen der regionalen Verteilung der Nährstoffe Silikat und Nitrat (KaLLE 1937) konnte das anomale Verhalten des Phosphats in der Konvergenzzone unterstützt werden. 
Enge Beziehungen zwischen physikalischen und geologischen Ergebnissen innerhalb der Deutschen Bucht sind nach Auffassung des Verfassers darin zu erblicken, daß der Kalkgehalt der Sedimente und des Oberflächenwassers durch einen Kreislauf bestimmt wird. Der von Pratje (1931) in einer Übersichtskarte mit der regionalen Kalk-

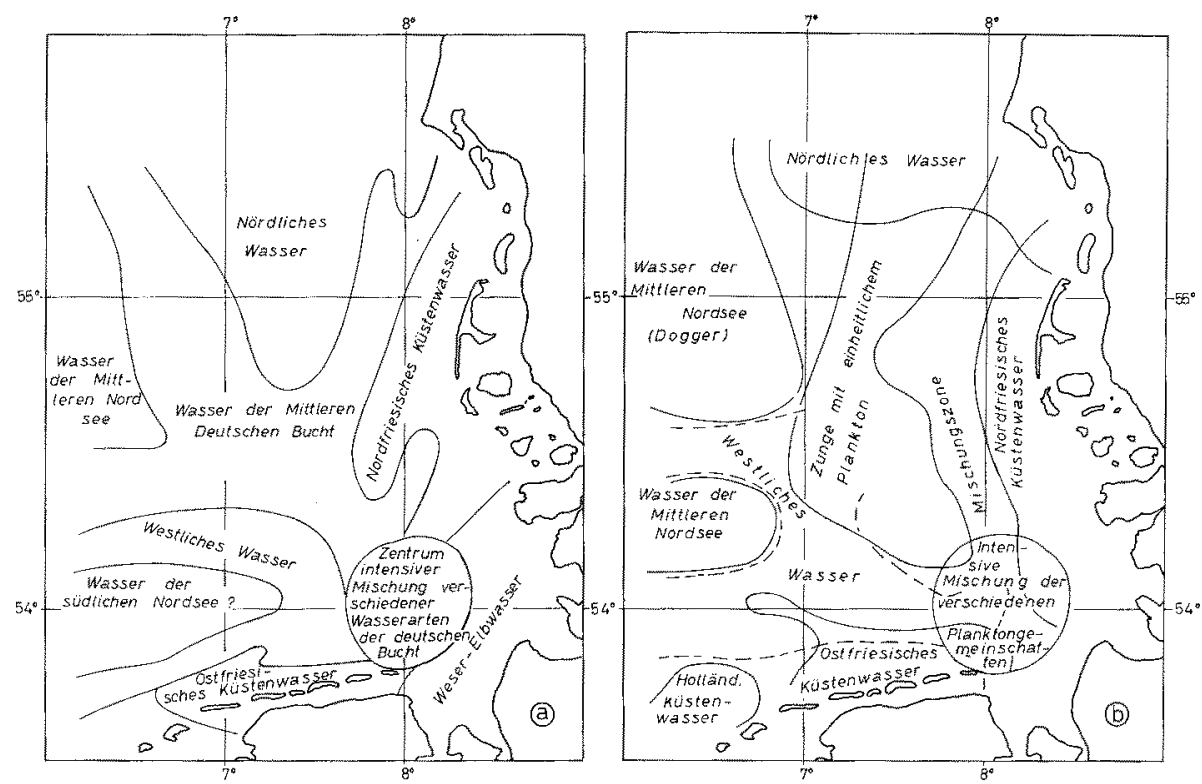

Abb. 18: Die sechs Wasserarten der Deutschen Bucht im Mai 1933 (a) auf Grund der $\triangle A$ Gehaltsbeobachtungen und (b) auf Grund von Planktonuntersuchungen. (Nach GoEDECKE 1936)

gehaltsverteilung der Sedimente bezeichnete sogenannte Mittelbezirk mit dem nordwärts gerichteten Ast, der das nördliche Schlickgebiet enthält, ist das Abbild der Zone mit dem Gehalt von anomalen suspendierten Kalkpartikelchen. Eine weitere Obersichtskarte mit der Verbreitung und Häufigkeit der Foraminiferen in den. Sedimenten der Deutschen Bucht unterstützen die Auffassung von dem Vorhandensein einer Konvergenzzone.

Zum Schluß soll in diesem Zusammenhang noch einmal auf die Lage des von SO nach NW sich erstreckenden Schlickstreifens am Boden der Deutschen Bucht hingewiesen werden. Die Zone des Schlickstreifens wird durch die Ausscheidungen der feinsten bisher noch in Suspension gehaltenen Partikelchen anorganischen Ursprungs bedingt. Von geologischer Seite gibt es allerdings Argumente, welche eine andere Erklärung für das Vorhandensein dieses Schlickstreifens heranziehen. Zwischen Bodenrelief und Konvergenzzone kann man vielleicht auch Beziehungen rein nach geographischen Gesichtspunkten ablesen. Es handelt sich hier um die "Kantenlage" der zyklonalen Wirbel in der Konvergenzzone. Die Lage der einzelnen Wirbel verläuft etwa auf einer Linie, welche durch ein Gebiet des Meeresbodens hindurchgeht, das vor der nordfriesischen Küste von der 30- und 35-m-Tiefenlinie begrenzt wird. 


\section{RUCKBLICK}

Wenn man die hier zusammengetragenen meereskundlichen Tatsachen aus den verschiedenen Disziplinen noch einmal überblickt, so lassen sie nach den bisherigen Ergebnissen den Schluß zu, daß man mit dem Vorhandensein einer Konvergenzzone in der Deutschen Bucht rechnen muß. Viele Fragen sind noch ungeklärt und bedürfen der weiteren Untersuchung. Vor allem muß jetzt das nächste Ziel die Darstellung einer Ubersichtskarte mit den Reststromverhältnissen in diesem Meeresgebiet der Nordsee sein. Wenn wir aber in diesem Zusammenhang das Problem der Verschmutzung in der Nordsee und insbesondere in der Deutschen Bucht aufgreifen wollen, so müssen wir, um diese schwerwiegende Frage beantworten zu können, die vorläufigen Ergebnisse bisheriger Konvergenzuntersuchungen als Grundlage mit heranziehen.

\section{ZUSAMMENFASSUNG}

1. Dynamisch gesehen ist die Konvergenz der Deutschen Bucht eine Frontalzone, in welcher zwei verschiedene Wasserkörper aufeinandertreffen und sich ïberlagern. Das spezifisch schwerere Nordseewasser schiebt sich unter das leichtere Küstenwasser. Die horizontale und vertikale Dichteverteilung in diesem Meeresgebiet bewirkt eine Asymmetrie in der Dauer von Flut und Ebbe sowie in derjenigen von Flut- und Ebbstrom. Im großen und ganzen gesehen läut die Ebbe bzw. der Ebbstrom länger als die Flut beziehungsweise der Flutstrom.

2. Auf Grund der $S^{0} / 00^{-}$und Dichte- $\left(\sigma_{t^{-}}\right)$Verteilung kann man Aussagen über das Bild der unperiodischen Wasserversetzungen (Restströmungen) machen. Entlang der Konvergenzzone bilden sich im Rahmen des großen zyklonen Wirbels der Deutschen Bucht mehrere Teilwirbel, welche auf einen starken Vermischungsprozeß zwischen den beiden Wasserkörpern schließen lassen.

3. Berechnungen von Stromversetzungen (des Massentransports) in der Oberflächenund Bodenschicht auf Grund der Dichteverteilung ergeben einen Dichtegradientstrom von der Größenordnung 10 bis $15 \mathrm{~cm} / \mathrm{sec}$ vor den Mündungstrichtern der großen Ströme Elbe und Weser sowie Grenzwerte von 4 bis $10 \mathrm{~cm} / \mathrm{sec}$ in der Konvergenzzone, damit höhere Werte als in den angrenzenden Bereichen. Die nordfriesische Seite zeigt höhere Geschwindigkeitswerte als die ostfriesische Seite.

4. Der horizontale und vertikale Aufbau von Temperatur und Salzgehalt in der Deutschen Bucht verursacht innerhalb der Konvergenzzone starke $\mathrm{t}^{0} \mathrm{C}-$ und $\mathrm{S}^{0} / 0^{-\mathrm{Gra}}$ dienten sowie markante jahreszeitliche Unterschiede im Verhalten von Temperatur und Salzgehalt entlang der ostfriesischen und nordfriesischen Küste.

5. Die Bedeutung der Konvergenzzone für den Stoffhaushalt dieses Meeresgebietes der Nordsee ist darin zu sehen, daß wahrscheinlich innige hydrographisch-chemische, hydrographisch-biologische und hydrographisch-geologische Zusammenhänge vorhanden sind.

6. Die Konvergenzzone der Deutschen Bucht ist - insbesondere im Frühjahr - ein Gebiet mit Sauerstoffübersättigung, in dem eine besonders intensive Planktonproduk- 
tion erfolgt. Mit ihren großen Salzgehaltsgradienten stellt sie eine Zone dar, in welcher verschiedene Nährstoffkomponenten wie Kalk, Nitrat, Phosphat, Silikat (Trübungsfaktor) angereichert werden.

7. Der Kalkgehalt des Oberflächenwassers der Deutschen Bucht steht im Kreislauf mit dem Kalkgehalt der Sedimente der Deutschen Bucht. Zwischen der Häufigkeitsverteilung der Foraminiferen, dem Vorhandensein des Schlickstreifens und der Kantenlage der hydrographischen Wirbel bestehen enge Beziehungen zur Konvergenzzone der Deutschen Bucht.

\section{ZITIERTE LITERATUR}

BöHnecke, G., 1922. Salzgehalt und Strömungen der Nordsee. Veröff. Inst. Meeresk. Univ. Berlin. (Geogr. nat. wiss. R. A.) 10, 1-34.

GоврескE, E., 1936. Der Kalkgehalt im Oberfächenwasser der Unterelbe und der Deutschen Bucht. Aus Arch. dt. Seew. 55 (1).

- 1939. Über unperiodische Wasserversetzungen in der Deutschen Bucht. Rapp. P. - v. Réun. Cons. perm. int. Explor. Mer 109 (3), 89-92.

- 1941. Beiträge zur Hydrographie der Konvergenz der Deutschen Bucht. Annln Hdrogr. Berl. 69 (11), $345 \mathrm{ff}$.

- 1952. Uber Intensität und Jahresgang der thermohalinen Schichtung in der Deutschen Bucht. Veröff. Inst. Meeresforsch. Bremerh. 1, 236-245.

- 1955. Uber die Intensität der Temperatur-, Salzgehalts- und Dichteschichtung in der Deutschen Bucht. Dt. bydrogr. Z. 8, 15-28.

- 1958a. Hydrographische Betrachtungen über die regionale Verteilung der mittleren Eisvorbereitungs- und Eisabschmelzzeit in der Deutschen Bucht und der westlichen Ostsee mit Karten. Dt. bydrogr. Z. 11, 1-22.

- 1958b. Uber Höhe und Eintrittszeit der Extreme sowie deren Schwankungen im mittleren Jahresgang von Temperatur, Salzgehalt und Dichte des Wassers in der Deutschen Bucht. Dt. hydrogr. Z. 11, 137-165.

- SMed, J. \& Tomczak, G., 1967. Monatskarten des Salzgehaltes der Nordsee, dargestellt für verschiedene Tiefenhorizonte. ErgänzHft. dt. bydrogr. Z. (R. B.) 9.

KaLLE, K., 1932. Phosphatgehaltsuntersuchungen in der Nord- und Ostsee im Jahre 1931. Annln Hydrogr. Berl. 60 (1), 6 ff.

- 1937. Nährstoffuntersuchungen als hydrographisches Hilfsmittel zur Unterscheidung von Wasserkörpern. Annln Hydrogr. Berl. 65 (1), 1 ff.

- 1943. Der Stoffhaushalt des Meeres. Geest \& Portig, Leipzig, 263 pp. (Probleme kosm. Phys. 23.)

- 1949. Die natürlichen Eigenschaften der Gewässer. In: Handbuch der Seefischerei Nordeuropas. Hrsg. von H. Lübbert, E. Ehrenbaum \& A. Willer. Schweizerbart, Stuttgart, 1 (1), 1-37.

Neumann, H. \& Meier, C., 1964. Die Oberflächenströme in der Deutschen Bucht. Dt. bydrogr. Z. 17, 1-40.

Wattenberg, H., 1941. Über die Grenzen zwischen Nord- und Ostseewasser. Annln Hydrogr. Berl. 69 (9), $265 \mathrm{ff}$.

WENDICKE, F., 1913. Hydrographische und biologische Untersuchungen auf den deutschen Feuerschiffen der Nordsee 1910/11. Die hydrographischen Ergebnisse. Veröff. Inst. Meeresk. Univ. Berl. 3, XII, 1-123; 1-94.

Zorell, F., 1935. Beiträge zur Hydrographie der Deutschen Bucht. Aus Arch. dt. Seew. 54 (1). 